\title{
PRESENT STATUS OF THE SYNCHROTRON RADIATION FACILITY NEWSUBARU
}

\author{
S. Hashimoto ${ }^{\dagger}$, Y. Shoji, Y. Fukuda, S. Miyamoto, M.Niibe and A. Ando, \\ NewSUBARU, 1-1-2 Koto, Kamigori, Akou, Hyogo 678-1205, JAPAN
}

\begin{abstract}
The synchrotron radiation facility NewSUBARU has been constructed in SPring-8 site and user experiments have been started since February 2000. The $1.5 \mathrm{GeV}$ electron storage ring with circumference of $119 \mathrm{~m}$ produces soft X-ray synchrotron radiation. The present status of the storage ring and the facility is presented.
\end{abstract}

\section{INTRODUCTION}

The synchrotron radiation (SR) facility NewSUBARU is a light source designed to generate bright light in the soft X-ray region. It is located in the SPring-8 site and consists of $1.5 \mathrm{GeV}$ electron storage ring, insertion devices and several beamlines.

In 1994 the Laboratory of Advanced Science and Technology for Industry (LASTI) [1] was founded to initiate the NewSUBARU project [2] at Himeji Institute of Technology. Hyogo prefecture supports the financial costs of both construction and operation of the facility, where research for industrial application of SR and support to company users who is not familiar with experiments using SR are important tasks in addition to basic research.

The construction of the facility began in December 1996 and was completed in August 1998. The beam commissioning started in September 1998 and the first SR was observed in the end of 1998. At the beginning of the beam commissioning, the dynamic gas load due to the SR-induced desorption was so large that the rapid beam loss due to the gas scattering made it difficult to end the commissioning in a reasonable period of time. During the summer shutdown of 1999 the vacuum system of insertion devices was upgraded to overcome this problem. As a result we succeeded in the maximum stored current of $100 \mathrm{~mA}$ and the beam lifetime was drastically improved [3]. Since February 2000 the user experiments using SR from bending magnets have been started and some excellent results have been obtained in research such as lithography.

\section{STORAGE RING}

The main parameters of the ring are summarized in Table I. The $1.5 \mathrm{GeV}$ electron storage ring of the NewSUBARU is racetrack-type with the circumference of $119 \mathrm{~m}$ and has two $14-\mathrm{m}$ and four $4-\mathrm{m}$ straight sections. Two 4-m straight sections are allocated for a RF

${ }^{\dagger}$ hashi@lasti.himeji-tech.ac.jp cavity and a septum magnet for beam injection. Four straight sections can be used for insertion devices. The long straight sections are one of unique features of this ring. The arrangement of the ring and beamlines are shown in Figure 1.

In addition to 12 normal bending magnets the ring adopts 6 inverse bending magnets in order to adjust momentum compaction factor between -0.001 to +0.001 . Small value of this factor makes it possible to shorten electron beam length and pulse duration of SR. During user time the ring is operated with momentum compaction factor of +0.001 . In machine study we demonstrated small momentum compaction factor of +0.0003 . The operation with negative momentum compaction factor has also been challenged.

The stable operation of the SPring- 8 linac and our enormous effort made it possible to achieve injection efficiency of over $90 \%$ from the linac to the storage ring. The maximum stored current of $100 \mathrm{~mA}$ is limited by regulation on radiation safety, although the designed value is $500 \mathrm{~mA}$.

The electron beam lifetime is determined by the scattering of electrons with residual gas molecules. The beam dose during one operation cycle (about three weeks) is only a few Ahrs because of the injection charge limit determined by the thin tunnel wall. The beam lifetime is about two hours with a stored current of $60 \mathrm{~mA}$. In order to improve the lifetime the vacuum system will be upgraded in summer shutdown of 2001.

Electron orbit is measured with 18 beam position monitors (BPMs) in vertical and horizontal planes. Closed orbit distortion (COD) is corrected within $20 \mu \mathrm{m}$ by 18 steering magnets.

Table I: Storage ring parameters

\begin{tabular}{ll}
\hline Circumference $(\mathrm{m})$ & 119 \\
Injection energy $(\mathrm{GeV})$ & 1.0 \\
Beam energy $(\mathrm{GeV})$ & 1.0 \\
Beam current $(\mathrm{mA})$ & 100 (Maximum) \\
& 60 (Top-up operation) \\
Beam lifetime (hour) & $2 @ 60 \mathrm{~mA}$ \\
Tunes & $6.23 / 2.17$ \\
Harmonic number & 198 \\
Natural Emittance (nm rad) & 40 \\
Coupling & $7 \%$ \\
Energy spread & $0.047 \%$ \\
Momentum compaction factor & +0.001 \\
\hline
\end{tabular}




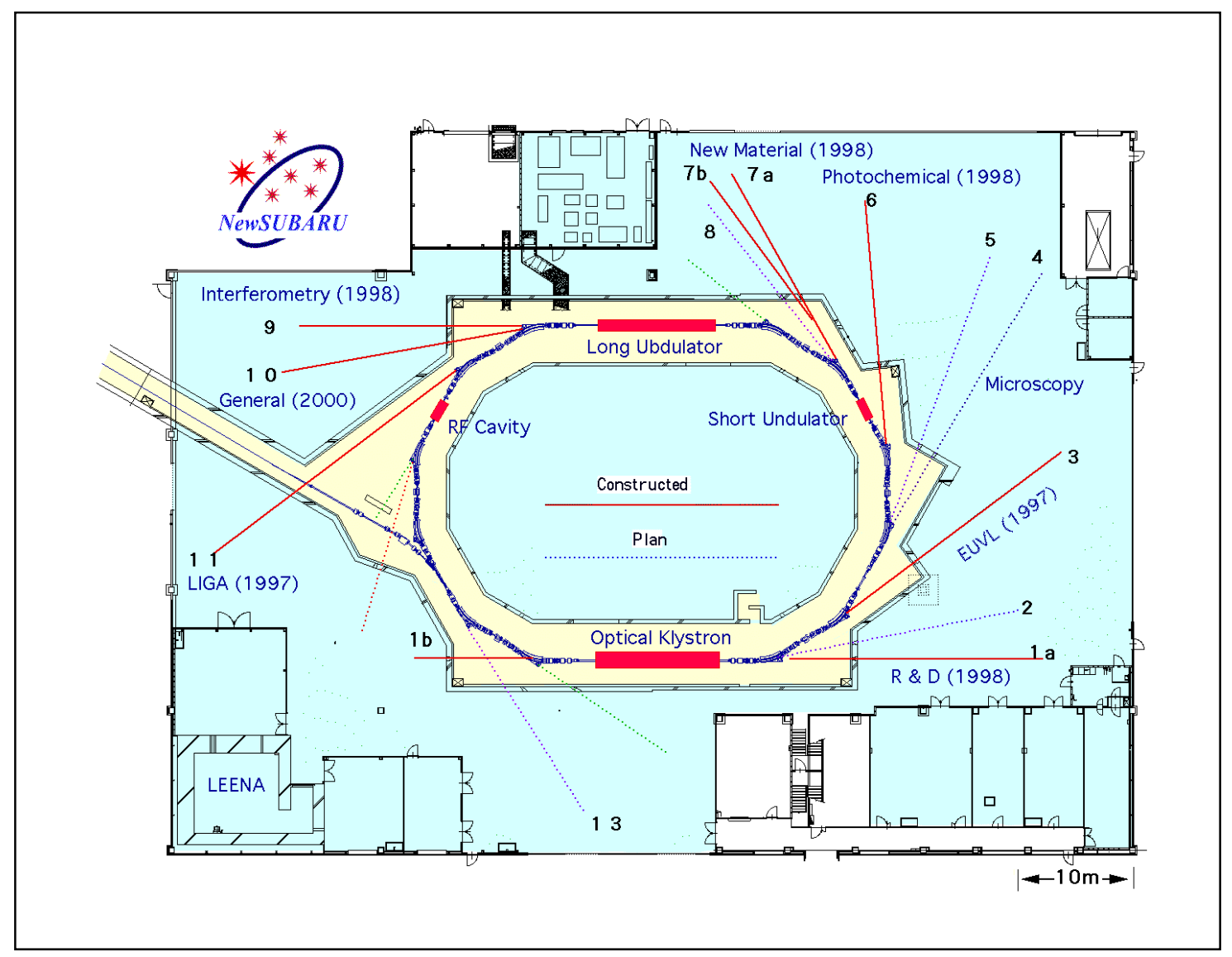

Figure 1: NewSUBARU storage ring and beamlines.

The electron beam emittance has been measured by direct observation of visible SR with CCD camera. The estimated emittance is in good agreement with designed value. The double sweep streak camera was also installed in order to measure bunch length of electron beam and is under calibration.

We have also observed ion trap instability. In order to avoid the instability, high voltage of $800 \mathrm{~V}$ is applied to ion clearing electrodes and the ring is operated with the filling pattern in which 50 bunches are sequentially filled. The beam lifetime is also improved by giving perturbation on electron beams in vertical direction with a RF shaker because ionized molecules stayed around an electron orbit are kicked away by the perturbation.

In the single bunch operation the blowup of the electron beam size caused by energy widening due to microwave instability was observed [4].

The operation energy of the ring has been restricted to $1.0 \mathrm{GeV}$ according to the regulation on radiation safety. The beam-energy ramping to $1.5 \mathrm{GeV}$ will be possible in 2001.

\section{INSERTION DEVICES}

Three insertion devices have been installed in the ring: 11-m Long Undulator (LU), Optical Klystron Free Electron Laser (OK-FEL) in 14-m long straight sections and Short Undulator (SU) in one of 4-m sections. The calculated on-axis brilliance is shown in Figure 2.

LU has 200 periods and is $10.8-\mathrm{m}$ long. Generally speaking, it is difficult to operate such a long insertion device in a relatively low energy storage ring. We have successfully demonstrated the operation of the storage ring with LU closed gap of $35 \mathrm{~mm}$, although the beam life is reduced to $70 \%$, however the measured spectrum width of undulator radiation was broader than calculated one. We suspect that there is horizontal error field in LU and electron orbit is vertically kicked. The fact that measured vertical distribution of total power of radiation was also broader than calculated one agrees with this assumption. Field measurement before installation of LU did not show such a large error. Error field may arise

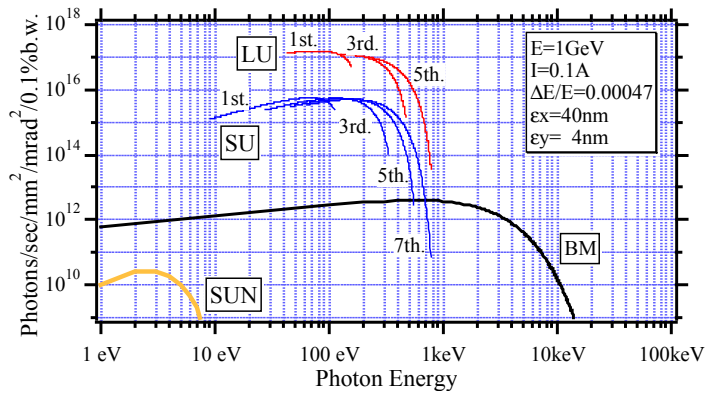

Figure 2: Light sources at NewSUBARU 
Table II Characteristics of beamlines at the NewSUBARU light source.

\begin{tabular}{llll} 
BL & SOURCE & ENERGY & PURPOSE \\
\hline 1 & Undulator (OK) & $0.006 \mathrm{KeV}$ & Light source R\&D, Free electron laser, Compton scattering \\
3 & Bending & $0.08-0.3 \mathrm{KeV}$ & Extreme Ultra Violet Lithography (EUVL) \\
4 (plan) & Bending & $0.3-0.6 \mathrm{KeV}$ & Development of equipment for medical diagnosis \\
6 & Bending & $<1 \mathrm{KeV}$ & Development of new material \\
7 & Undulator (SU) & $<1 \mathrm{KeV}$ & Structural analysis, Material development \\
9 & Undulator (LU) & $0.08-0.3 \mathrm{KeV}$ & High precision measurement \\
10 & Bending & $<1 \mathrm{KeV}$ & Multi-purpose BL \\
11 & Bending & $<6 \mathrm{KeV}$ & Micro system technology (LIGA) \\
\hline
\end{tabular}

from field measurement error, sunk of concrete floor, distortion of girder, shimming and so on. In order to withdraw the full performance of LU, re-measurement of magnetic field and re-alignment are performed in the summer of 2001.

FEL is a tunable light source in which electromagnetic field stored in a resonator is amplified by a relativistic electron beam. OK undulator is made from normal electromagnets with periods of 160 or $320 \mathrm{~mm}$ and was designed to oscillate at relatively long wavelength from visible to infrared region. The spontaneous emission from OK-FEL at visible wavelength was measured in October 2000 and FEL oscillation experiments started in 2001.

\section{BEAMLINE AND USER EXPERIMENTS}

There are 13 beamports in the ring, 4 for insertion devices and 9 for bending magnets. Seven beamlines are completed in June 2001. The characteristics of these beamlines are shown in Table II.

There are three modes of machine operations: user experiment, machine study and vacuum baking by SR. The ring now operates 24 hours a day and 5 days a week. In principle 9:00-17:00 from Tuesday to Friday is user time for SR experiments and Monday for machine study. In order to keep the stored beam current $60 \mathrm{~mA}$ during user time, the top-up operation is performed, that is, electron beams are continuously injected from linac to the ring every 60 seconds. From 17:00 to 9:00 the ring is operated, even though there is no user experiment or machine study, in order to gain beam dose.

With an increase of beam dose on-beam pressure decreases and beam lifetime is improved. Although the beam lifetime is increasing day by day, lots of beam dose is required in order to realize long lifetime.

From January to November 2000 the total operation time is $2473 \mathrm{hrs}$ of which 776hrs for the user experiments, $297 \mathrm{hrs}$ for the machine study and $1400 \mathrm{hrs}$ for vacuum baking by SR. The machine availability is about $97 \%$. The machine fault of $3 \%$ is due to the troubles of power supplies of the bending magnets and those of klystron.
The number of facility users reaches over 50 in June 2001 and is expected to be rapidly increasing. Researchers who want to perform experiments with SR can use the facility under a joint research with LASTI.

\section{SUMMARY}

The synchrotron radiation facility NewSUBARU was constructed in 1998 with support from SPring-8. The user experiments have been started since February 2000. Seven beamlines are completed and some excellent experimental results have been obtained. The beam lifetime is only a few hours but is gradually improving with an increase of beam dose.

The authors thank all SPring-8 staff for their cooperation.

\section{REFERENCES}

[1] http://www.lasti.himeji-tech.ac.jp/Index.html [2] A. Ando, S. Amano, S. Hashimoto, H. Kinoshita, S. Miyamoto, T. Mochizuki, M. Niibe, M. Terasawa and T. Watanabe, Proc. IEEE Particle Accelerator Conf. 1997 757-759 (1997)

[3] S. Hashimoto et al, Proceedings of SRI2000, Nucl. Instrum. \& Meth. A, in press.

[4] A. Ando, Y. Fukuda and S. Hashimoto, "Beam Size Blowup and Energy Widening in NewSUBARU", Nucl. Instrum. \& Meth. A, in press. 